\title{
Compliance with RSV prophylaxis: Global physicians' perspectives
}

\author{
This article was published in the following Dove Press journal: \\ Patient Preference and Adherence \\ 9 July 2009 \\ Number of times this article has been viewed
}

\section{Kari S Anderson \\ Victoria M Mullally \\ Linda M Fredrick \\ Andrew L Campbell}

Abbott Laboratories, Abbott Park, IL, USA
Correspondence: Kari S Anderson I 75 Chandler Drive,Westminster, MD, 21157, USA

Tel + I 4I0 3860985

Email kari.anderson@abbott.com
Abstract: Respiratory syncytial virus (RSV) is a significant cause of morbidity in high-risk infants. Palivizumab is proven to prevent serious RSV disease, but compliance with prophylaxis (monthly doses during the RSV season) is essential to ensure protection. We invited 453 pediatricians to participate in a survey to identify their perspectives of barriers to compliance and interventions to improve compliance with palivizumab prophylaxis schedules. One hundred physicians from five continents completed the survey, identifying caregiver inconvenience, distance to clinic, cost of prophylaxis, and lack of understanding of the severity of RSV as the most common reasons for noncompliance. They recommended provision of educational materials about RSV, reminders from hospital or clinic, and administration of prophylaxis at home to increase compliance. Globally, physicians recognize several obstacles to prophylaxis compliance. This survey suggests that focused proactive interventions such as empowering caregivers with educational materials and reducing caregiver inconvenience may be instrumental to increase compliance.

Keywords: medication adherence, respiratory syncytial virus infections, infant, premature, immunization, passive

Adherence to health care interventions is an important part of enhancing health. Nonadherence to intervention, be it pharmaceutical or surgical treatment, physical therapy, dietary changes, lifestyle changes, screening procedures, vaccinations, or any other regimen, has significant consequences for patients in terms of outcome and quality of life, as well as a significant cost and resource burden on the health care system.

The World Health Organization (WHO), in its report on adherence to medications, defined adherence to long-term therapy as: "The extent to which a person's behaviour ... corresponds with agreed recommendations from a health care provider."' The definition recognizes the partnership between patients and providers in making health care choices, in which the patient is an active participant in decisions and not a passive recipient of instructions from the physician or other health care provider. While this definition was adopted in the context of treatment for chronic conditions, it is also applicable to surveillance activities, such as routine screening procedures, or preventive measures, such as vaccination schedules.

The WHO report and much of the literature describe adherence related to chronic disease, but nonadherence to disease prophylaxis regimens is also of concern. The economic burden caused by nonadherence to prophylaxis against many preventable infectious diseases can be estimated, but it is less straightforward to estimate costs 
associated with partial adherence, for example to some but not all vaccines or to some but not all doses of an individual vaccine.

Respiratory syncytial virus (RSV) infection is the leading cause of lower respiratory tract infection (LRTI) in infants. ${ }^{2} \mathrm{RSV}$ is a seasonal virus in most regions of the world. Epidemics lasting 4-6 months occur during the winter season in temperate climates, with peak infection periods in December and January in the northern United States, Canada, and much of northern Europe. ${ }^{3}$ By the age of two years, nearly all children have been infected. ${ }^{2}$ Generally, RSV infection results in an upper respiratory tract infection; however, $25 \%$ to $40 \%$ of infected children develop a mild-to-moderate LRTI, and about $1 \%$ of previously healthy infected children require hospitalization. Risk factors contributing to serious RSV disease and hospitalization include chronic lung disease, congenital heart disease, and premature birth ( $\leq 35$ weeks gestational age). ${ }^{3,4}$ Greenough $^{5}$ and Sampalis ${ }^{6}$ demonstrated that RSV hospitalization is associated with greater utilization of health care resources in infants $\leq 35$ weeks of gestation. RSV-LRTI and associated hospitalizations pose a significant burden of illness to patients and their families and have an economic impact on the health care system. ${ }^{7}$ Prevention of RSV-LRTI may thus reduce this burden to families and society in general.

Because no licensed vaccines are currently available to prevent RSV infection, passive immunoprophylaxis with anti-RSV IgG antibody is the only option for preventing RSV disease in high-risk children. Two passive immunoprophylaxis agents are approved for the prevention of RSV infection. Palivizumab (marketed in the US by MedImmune, LLC, Gaithersburg, MD; marketed outside the US by Abbott, Abbott Park, IL) is a humanized immunoglobulin G ( $\mathrm{IgG}$ ) monoclonal antibody approved for use in infants at high risk for severe RSV disease, and is administered monthly by intramuscular injection throughout the RSV season. Intravenous RSV immunoglobulin (RSV-Ig) is prepared from pooled human blood, and is administered monthly by intravenous (IV) infusion during the RSV season. Guideline recommendations for RSV prophylaxis in high-risk infants have been published by the American Academy of Pediatrics, ${ }^{8}$ the Canadian Paediatric Society, ${ }^{9}$ and other organizations dedicated to children's health and disease prevention. Though both palivizumab and RSV-Ig are included in guideline recommendations, ${ }^{8,9}$ palivizumab is the preferred agent for RSV prophylaxis; not only is it effective, it is also convenient to administer, leading to significantly reduced time costs for patients' families. ${ }^{10}$ Intramuscular injection is completed in a matter of minutes, compared with several hours required to complete IV infusion. In addition, IM injection is a simple procedure that can be performed by medical office staff, whereas IV infusion requires specialized training and equipment.

Palivizumab is not a vaccine. It provides passive immunization rather than eliciting an active immune response against RSV, ${ }^{4}$ and must be administered monthly during the RSV season in order to be effective. Palivizumab prophylaxis depends upon full compliance with the monthly dosing schedule. However, full compliance rates vary in different regions studied. In developed countries, rates between $36 \%$ and $98 \%$ have been reported, ${ }^{11-13}$ with differences depending not only on the region studied but also on where the doses are delivered (eg, central hospital, pediatrician's office, or at a patient's home).

As suggested by the widely varying rates reported, there are many barriers to full compliance with palivizumab prophylaxis. For example, Langkamp ${ }^{14}$ surveyed parents at a single center in the United States to identify barriers to full compliance and discovered that a key factor in compliance was parental belief in the benefit of palivizumab. Difficulty in transportation to the hospital to receive injections also was identified as a compliance barrier in that survey. Though not a significant factor in the Langkamp report, many parents were concerned about out-of-pocket costs of the drug and other indirect costs (eg, time spent negotiating with insurers). Bracht and colleagues ${ }^{11}$ established an RSV prevention program at three tertiary centers and the surrounding areas in Canada. Education of parents about risks of RSV disease and benefits of prophylaxis with palivizumab were key components of the program, which achieved 98\% compliance. Cost of the treatment was not a factor for this Canadian population, but the researchers identified language barriers and educational limitations in some families as obstacles to the full understanding of the instructional materials provided. Similarly, Pignotti ${ }^{15}$ identified language barriers which caused difficulty in communicating the severity of RSV and the proposed prophylactic schedule as being a key component of nonadherence to the full palivizumab dosing schedule in Italy. Singleton and colleagues ${ }^{16}$ reported that transportation delays from adverse weather conditions contribute to low compliance with recommended palivizumab administration in an Alaskan Native population. Compliance increased when palivizumab was delivered by a local trained health aide. ${ }^{16}$ Thus, previous work has revealed that a lack of parental understanding of RSV disease and prevention, communication obstacles, and transportation difficulties 
are important barriers to achieving full compliance with palivizumab prophylaxis.

We surveyed physicians to discover if their perceptions of barriers to full compliance with palivizumab prophylaxis matched those reported by patients' families and in the literature. Pediatricians from countries in which palivizumab is approved for use were identified by Abbott medical directors and staff and invited by electronic mail to participate anonymously in an internet-based survey (http://www. markettools.com/). Physicians attending an invitational global medical conference were invited to participate in the survey at on-site computer terminals. There was no duplication of invitations; physicians who attended the conference were not contacted by electronic mail to complete the survey. In all, 453 physicians were invited to respond to the survey questions. Some invited physicians prescribed palivizumab and some did not. The survey was provided in English and contained 29 questions to assess physician practice habits, perceptions of obstacles to compliance, and measures to enhance compliance with palivizumab prophylaxis (Table 1). One hundred physicians (response rate $=22 \%$ ) completed the survey from Europe $(n=60)$, Asia $(n=15)$, North America ( $\mathrm{n}=13$; Canada and Mexico only), South America $(\mathrm{n}=7)$, and Africa $(\mathrm{n}=5)$. Information about the responding physicians was based on their answers to survey questions. No information about nonresponders was collected.

The physicians responding to our survey identified inconvenience to parents and distance to the clinic, cost of palivizumab, and a lack of understanding of the threat of RSV as the primary barriers to adherence to the full dosing schedule (Figure 1). The most frequent barriers identified differed when the responses were stratified by region. For example, European physicians $(n=60)$ believed that inconvenience and distance to the clinic were the most important barriers to full compliance, whereas cost of prophylaxis was less important. By contrast, non-European physicians $(n=40)$ ranked cost as the primary barrier to full compliance with palivizumab prophylaxis, though inconvenience to caregivers was also thought to be important. Our survey asked physicians to speculate about the reasons their patients' parents might be noncompliant with palivizumab dosing schedules, in contrast to other surveys in which parents were asked directly. It is interesting that the physicians in our survey identified barriers to adherence similar to those that have been reported from the perspectives of patients' parents.

In our survey, physicians recommended additional educational materials and education of patients' families about the threat from severe RSV disease, frequent reminders from the hospital, and administration of palivizumab in patients' homes as key drivers of full compliance (Figure 2). The same drivers were identified when the responses were stratified by region (Europe versus non-Europe). Thus, our survey results emphasize the universal importance of educating families about RSV disease and palivizumab prophylaxis. In addition, the results support home administration of palivizumab as a driver for full compliance with the monthly dosing schedule, in agreement with previous work that has demonstrated higher compliance rates with home or local administration. ${ }^{12,16}$

Of the physicians responding to our survey, $68 \%$ believe that their patients' parents think that palivizumab is a vaccine. In fact, $38 \%$ of the responding physicians believe that palivizumab is a vaccine. Thus, our survey identified a need for further education not only of patients' families, but also in some cases of physicians themselves. Unlike most vaccines, palivizumab has a strict dosing schedule that must be followed to achieve efficacy throughout the RSV season. Equating palivizumab to a vaccine may cause caregivers or even physicians to falsely believe that a single injection will protect at-risk children. In our survey, $86 \%$ of the respondents provide information to their patients' caregivers about RSV and prophylaxis. Physicians need to be fully educated about the action of palivizumab and the necessity of multiple doses on a strict schedule in order to effectively communicate the importance of adherence to their patients' families.

Though the survey invited a large number of physicians to participate, the results are limited by bias in the selection process and the low rate of response. Invitations to participate were not random, because contact information for a random sample of pediatricians in each country was unavailable. In addition, responders were more likely to be fluent in English than nonresponders, since the survey was provided in English. On the other hand, physicians practicing in developed as well as developing countries participated in the survey, leading to a broad spectrum of responses. Furthermore, the anonymity of the survey may have encouraged responders to answer the questions candidly, contributing some strength to the results. Indeed, the barriers to compliance and the recommendations to increase compliance identified in this survey are similar to those previously reported in the literature, suggesting that the results may be generally applicable in a larger and more randomly selected population of pediatricians.

Infants are a special subpopulation in whom compliance to any medical regimen is dependent on parental decisions. Serious RSV disease resulting in hospitalization places a burden on families and on the health care system. 
Table I Survey questions

\begin{tabular}{ll}
\hline Number & Question \\
\hline I & Please select the country in which you currently practice \\
2 & Please select your specialty:
\end{tabular}

- With hemodynamically significant congenital heart disease?

Answers/Options

Drop-down list

- Pediatrics

- GP

- Family medicine

- Internal medicine

- Combined internal medicine/Pediatrics

- Surgery

- Other, please specify

- Neonatology

- Pediatrics

- Pulmonology

- Cardiology

- Infectious disease

- Neurology

- Immunology/Allergy

- Intensive care

- Other, please specify

$-<5$

- 5-10

- II-20

- 21-30

$-31-40$

$->41$

- Academic

- Private

- Government

- Other, please specify

- University

- Journals

- Medical representatives

- Medical or scientific memberships

- Attendance at congresses or conferences

- Other, please specify

- Highly educated (university)

- Some education (primary and secondary)

- No formal education

- Self-pay

- Government sponsored

- Private insurance

- Other, please specify

Respondent fill-in

The following four (4) questions will refer to specific patient types. For simplification purposes, they will be coded as shown below:

$<29$ wGA, no BPD: $<29$ weeks gestational age without bronchopulmonary dysplasia (BPD) or chronic lung disease (CLD)

29-32 wGA, no BPD: 29-32 weeks gestational age without BPD/CLD

33-35 wGA, no BPD: 33-35 weeks gestational age without BPD/CLD

BPD/CLD: diagnosed with BPD/CLD

CHD: diagnosed with congenital heart disease (CHD) 
Table I (Continued)

\begin{tabular}{ll}
\hline Number & Question \\
\hline 10 & Which of the following patient types do you see/treat in your practice? Se \\
& all that apply \\
& \\
& Please indicate your satisfaction with the literature supporting the use of \\
& palivizumab/Synagis ${ }^{\circledR}$ in the following high-risk groups. \\
& $-<29$ wGA, no BPD: \\
& $-29-32$ wGA, no BPD: \\
& $-33-35$ wGA, no BPD: \\
& - BPD/CLD: \\
& - CHD:
\end{tabular}

\section{Answers/Options}

- $<29$ wGA, no BPD

- 29-32 wGA, no BPD

- 33-35 wGA, no BPD

- BPD/CLD

- CHD

- None of the above

Respondent fill-in using I-5 scale $(I=$ extremely satisfied $-5=$ extremely dissatisfied)

12

In what percent of patients do you recommend palivizumab/Synagis ${ }^{\circledR}$

Respondent fill-in prophylaxis:

- $<29$ wGA, no BPD:

- 29-32 wGA, no BPD:

- 33-35 wGA, no BPD:

- BPD/CLD:

- CHD:

Palivizumab/Synagis ${ }^{\circledR}$ is indicated for the prevention of serious lower respiratory tract disease requiring hospitalization caused by respiratory syncytial virus (RSV) in children at high risk for RSV disease:

- Children born at 35 weeks of gestation or less and less than six months of age at the onset of the RSV season

- Children aged less than two years and requiring treatment for bronchopulmonary dysplasia within the last six months

- Children aged less than two years and with hemodynamically significant congenital heart disease

13
Have you ever NOT recommended palivizumab for a patient who met indications in the following patient groups?

- $<29$ wGA, no BPD

- 29-32 wGA, no BPD

- 33-35 wGA, no BPD

- BPD/CLD

- CHD

- Other, please specify

Please select the top three (3) reasons why you would NOT recommend palivizumab/Synagis ${ }^{\circledR}$ for your indicated patients.

Are parents/caregivers of patients provided with information on the risks of severe RSV and palivizumab prophylaxis?

Please indicate in which format the information is provided. Select all that apply.

Who is primarily responsible for providing the information to the patient's parent/caregiver?
Yes/No

- Lack of reimbursement

- Lack of data to support use

- Safety

- Cost of palivizumab

- Inconvenience for the patient/family

- Inconvenience for the medical staff/nursing staff

- Other, please specify

Yes/No

- Written (text only)

- Pictures/diagrams (visuals with text)

- Verbal

- Other, please specify

- MD

- Resident or fellow

- Nurse

- Office staff

- Other, please specify 
Table I (Continued)

\begin{tabular}{lll}
\hline Number & Question & Answe \\
\hline 18 & Is written information provided in the parent/caregiver's native language? & Yes/No \\
19 & For those who are not fluent in the local language, is an interpreter used? & Yes/No \\
20 & $\begin{array}{l}\text { Are parents/caregivers requested to repeat the information provided to them } \\
\text { in order to validate the teaching message? }\end{array}$ & Yes/No
\end{tabular}

21 When is RSV generally first discussed with your patients' parents/caregivers?

In patient populations where the parent/caregiver refuses initiation of prophylaxis, what do you believe are the top three (3) factors that contribute to a parent/caregiver's refusal?
What do you believe are the top three (3) factors that contribute most to noncompliance with palivizumab/Synagis ${ }^{\circledR}$ recommendations? (Noncompliance is defined as infants who have therapy initiated, but do not receive all recommended doses.)
Answers/Options

Yes/No

- Prior to primary hospital discharge (ie, birth hospitalization)

- After primary hospital discharge

- During hospital re-admission

- Never

- Other, please specify

- Inconvenience

- Cost

- Perceived lack of benefit from palivizumab/Synagis ${ }^{\circledR}$

- Incomplete knowledge of the threat from RSV

- Palivizumab/Synagis ${ }^{\circledR}$ perceived as unsafe

- Parent/caregiver's belief that RSV exposure can be limited

- Personal "antivaccine" beliefs

- Cultural beliefs

- Religious beliefs

- Other, please specify

- Inconvenience to parents/ caregivers

- Distance to clinic

- Parent/caregiver time off work

- Cost of product

- Issues related to other children

- Fear of injections

- Lack of availability of palivizumab

- Lack of perceived benefit of palivizumab by the parent/ caregiver

- Perception by parent/ caregiver that the product is unsafe

- Adverse reactions from palivizumab

- Adverse reactions from other medications or vaccines

- Lack of understanding regarding threat of RSV

- Other, please specify 
Table I (Continued)

\begin{tabular}{ll}
\hline Number & Question \\
\hline 24 & $\begin{array}{l}\text { What do you believe are the top three (3) factors that contribute most to full } \\
\text { compliance with palivizumab/Synagis }{ }^{\circledR} \text { recommendations? (Full compliance is } \\
\text { defined as patients receiving all recommended doses.) }\end{array}$
\end{tabular}

In order to increase compliance, what are the top three (3) interventions you would recommend?
Considering your country's routine immunization schedule, what $\%$ of children in your practice receive all recommended doses of immunizations? If you do NOT intend to recommend palivizumab to a patient who meets the

\section{Answers/Options}

- Educational materials regarding threat of RSV

- RSV illness in a previous child

- Recommendations from physician for palivizumab

- Administration of palivizumab in a previous child

- Coordination of palivizumab doses with other medical or vaccination visits

- Prearranged transportation to palivizumab administration visits

- Participation in parent or advocacy groups

- Reminders of the palivizumab administration visit from the hospital or clinic

- Administration of palivizumab in home

- Home educational and hospital follow-up visits from nurses

- Home educational and hospital follow-up visits from physicians

- Other, please specify

- Additional educational materials

- Satellite clinics

- Frequent reminders from the hospital

- Addition of home visits

- Home administration of palivizumab prophylaxis

- Education of patient's family

- Reimbursement

- Transportation to the palivizumab dosing visits

- Other, please specify

- $0 \%-25 \%$

- $26 \%-50 \%$

- $51 \%-75 \%$

- $76 \%-100 \%$

- My country does not have a routine immunization schedule

Yes/No

Yes/No

Yes/No indications for prophylaxis, would you provide information about RSV to the parents/caregivers? 
Inconvenience to parents/caregivers

Distance to clinic

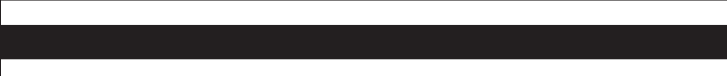

Cost of product

Lack of understanding regarding threat of respiratory syncytial virus

Lack of perceived benefit of palivizumab by the parent/caregive

Fear of injections

Parent/caregiver time off work

Lack of availability of palivizumab
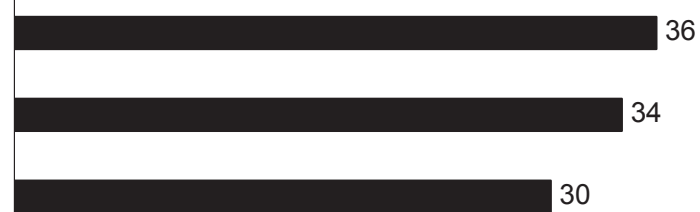

Adverse reactions from other medications or vaccines

Issues related to other children

Adverse reactions from palivizumab

Perception by parent/caregiver that the product is unsafe

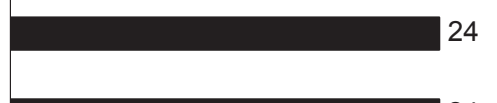

24

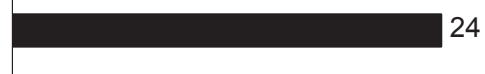

24
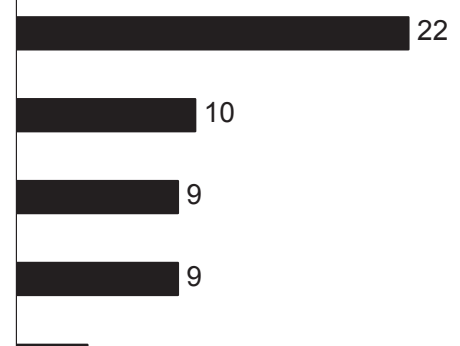

10

Figure I Barriers to full compliance. Physicians were asked what they believed to be the top three factors that contribute most to noncompliance with recommended palivizumab dosing schedules.

Additional educational materials

Frequent reminders from the hospital

Education of patient's family

Home administration of palivizumab prophylaxis

Reimbursement
Satellite clinics

Transportation to the palivizumab dosing visits

Other

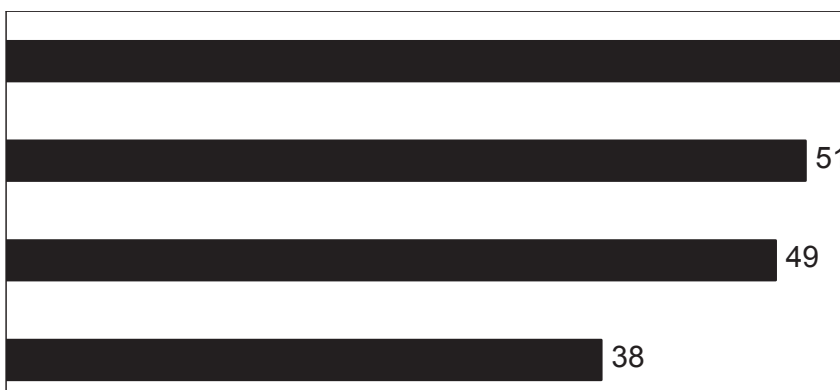

30
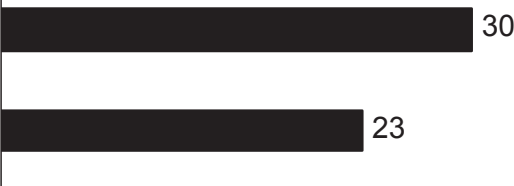

18

13

2

Figure 2 Recommended interventions. Physicians were asked to choose the top three interventions they would recommend to increase full compliance with palivizumab prophylaxis. 
Palivizumab prophylaxis has been shown to reduce the incidence of RSV-LRTI and hospitalization in at-risk infants, ${ }^{4,17}$ but effective prophylaxis requires full compliance with the monthly dosing schedule. While several approaches to increasing full compliance with RSV prophylaxis may be beneficial, including cost reductions (in some areas of the world), frequent reminders, home or local administration, and assistance with transportation to clinics, it is critically important that parents of children at high risk for severe RSV disease be empowered with clear information about the threat from RSV and the benefits of palivizumab prophylaxis to enable them to make informed choices. Physicians and other health professionals are primary sources of information for patients or their caregivers, and thus play a key role in guiding families in their choices about RSV prophylaxis. The physicians we surveyed recognized both the importance of educating families about RSV and prophylaxis, and their own roles in providing this information.

\section{Acknowledgments}

The authors would like to thank Laurinda Cooker, $\mathrm{PhD}$, of Abbott, for her assistance in the writing and preparation of this manuscript. The authors report no conflicts of interest in this work.

\section{References}

1. World Health Organization. Adherence to long-term therapies. Evidence for action. Geneva, Switzerland: World Health Organization; 2003.

2. Glezen WP, Taber LH, Frank AL, Kasel JA. Risk of primary infection and reinfection with respiratory syncytial virus. Am J Dis Child. 1986;140(6):543-546.

3. Law BJ, Carbonell-Estrany X, Simoes EA. An update on respiratory syncytial virus epidemiology: a developed country perspective. Respir Med. 2002;96(Suppl B):S1-S7.
4. Palivizumab, a humanized respiratory syncytial virus monoclonal antibody, reduces hospitalization from respiratory syncytial virus infection in high-risk infants. The IMpact-RSV Study Group. Pediatrics. 1998;102(3 Pt 1):531-537.

5. Greenough A, Cox S, Alexander J, et al. Health care utilisation of infants with chronic lung disease, related to hospitalisation for RSV infection. Arch Dis Child. 2001;85(6):463-468.

6. Sampalis JS. Morbidity and mortality after RSV-associated hospitalizations among premature Canadian infants. J Pediatr. 2003; 143(Suppl 5):S150-S156.

7. Pelletier AJ, Mansbach JM, Camargo CA Jr. Direct medical costs of bronchiolitis hospitalizations in the United States. Pediatrics. 2006; 118(6):2418-2423.

8. American Academy of Pediatrics Committee on Infectious Diseases and Committee on Fetus and Newborn. Revised indications for the use of palivizumab and respiratory syncytial virus immune globulin intravenous for the prevention of respiratory syncytial virus infections. Pediatrics. 2003;112(6 Pt 1):1442-1446.

9. Canadian Paediatric Society. Palivizumab and respiratory syncytial virus globulin intravenous for the prophylaxis of respiratory syncytial virus infection in high risk infants. Infectious Diseases and Immunization Committee and Fetus and Newborn Committee. Paediatr Child Health. 1999:4474-4480.

10. Robbins JM, Tilford JM, Gillaspy SR, et al. Parental emotional and time costs predict compliance with respiratory syncytial virus prophylaxis. Ambul Pediatr. 2002;2(6):444-448.

11. Bracht M, Heffer M, O'Brien K. Development, implementation, and evaluation of a community- and hospital-based respiratory syncytial virus prophylaxis program. Adv Neonatal Care. 2005;5(1):39-49.

12. Golombek SG, Berning F, Lagamma EF. Compliance with prophylaxis for respiratory syncytial virus infection in a home setting. Pediatr Infect Dis J. 2004;23(4):318-322.

13. Paul DA, Leef KH, Chidekel A, et al. Home delivery of palivizumab: outcomes and compliance in regional preterm infants. Del Med J. 2002;74(1):11-15.

14. Langkamp DL, Hlavin SM. Factors predicting compliance with palivizumab in high-risk infants. Am J Perinatol. 2001;18(6):345-352.

15. Pignotti MS, Catarzi S, Donzelli G. A 4-year survey on palivizumab respiratory syncytial virus (RSV)-prophylaxis: how can compliance be improved? J Matern Fetal Neonatal Med. 2006;19(4):221-224.

16. Singleton RJ, Bruden D, Brooks L, et al. Closer to home: local care improves compliance with RSV prophylaxis in high-risk infants. Int J Circumpolar Health. 2006;65(1):4-7.

17. Oh PI, Lanctôt KL, Yoon A, et al. Palivizumab prophylaxis for respiratory syncytial virus in Canada: utilization and outcomes. Pediatr Infect Dis J. 2002;21(6):512-518.
Patient Preference and Adherence

\section{Publish your work in this journal}

Patient Preference and Adherence is an international, peer-reviewed, open access journal that focusing on the growing importance of patient preference and adherence throughout the therapeutic continuum. Patient satisfaction, acceptability, quality of life, compliance, persistence and their role in developing new therapeutic modalities and compounds to

\section{Dovepress}

optimize clinical outcomes for existing disease states are major areas of interest. This journal has been accepted for indexing on PubMed Central. The manuscript management system is completely online and includes a very quick and fair peer-review system. Visit http://www.dovepress.com/ testimonials.php to read real quotes from published authors. 